\title{
Operational planning of large-scale energy systems with high share of renewable generation
}

This paper was downloaded from TechRxiv (https://www.techrxiv.org).

\section{LICENSE}

CC BY 4.0

SUBMISSION DATE / POSTED DATE

$11-06-2020$ / 05-02-2021

\section{CITATION}

Gea Bermúdez, Juan; Das, Kaushik; Koivisto, Matti Juhani; koduvere, Hardi (2020): Operational planning of large-scale energy systems with high share of renewable generation. TechRxiv. Preprint. https://doi.org/10.36227/techrxiv.12465230.v2

$\mathrm{DOI}$

10.36227/techrxiv.12465230.v2 


\title{
Operational planning of large-scale energy systems with high share of renewable generation
}

\author{
Juan Gea-Bermúdez, Kaushik Das, Senior Member, IEEE, Hardi Koduvere, and Matti Koivisto
}

\begin{abstract}
This paper proposes a mathematical model to perform optimal operational planning of large-scale energy systems with high share of renewable energy. Furthermore, it analyses the influence of different unit commitment modelling approaches on the operational planning outcomes. The value of co-optimisation of electricity and heating sector is emphasized in this paper. The results show the influence of massive renewable penetration in the energy sector towards 2050, and how this influences generation from other sources such as thermal and hydro. Including unit commitment constraints with integer variables leads to more realistic behaviour of the units, at the cost of increasing considerably the computational time. Relaxing integer variables reduces significantly the computational time, without highly compromising the accuracy of the results. Neglecting the unit commitment constraints leads to inaccurate results in terms of underestimation of costs, curtailment, wind's and solar PV's average revenue per energy unit sold, price volatility, and to overestimation of the flexibility of thermal units. Hence, depending on the purpose of the analysis, it is recommended to consider carefully the choice of unit commitment modelling approach and acknowledge the limitations. When the focus is on prices and revenues, using unit commitment constraints with integer variables is preferable.
\end{abstract}

Index Terms-Energy System, Large Scale, Optimisation, Operational Planning, Unit Commitment

\section{NOMENCLATURE}

$\begin{array}{cl}\text { Acronyms } & \\ V R E & \text { Variable Renewable Energy } \\ C H P & \text { Combined Heat and Power } \\ P 2 H & \text { Power-to-Heat } \\ D A & \text { Day Ahead } \\ U C & \text { Unit Commitment } \\ M I P & \text { Mixed Integer Linear Programming } \\ R M I P & \text { Relaxed Mixed Integer Linear Programming }\end{array}$

Sets

$\begin{array}{ll}g \in \mathbb{G} \mathbb{G} \mathbb{G} & \text { Generation and pure storage units } \\ y \in \mathbb{Y} & \text { Years } \\ s \in \mathbb{S} & \text { Seasons } \\ t \in \mathbb{T} & \text { Time steps } \\ a \in \mathbb{A} & \text { Areas } \\ r \in \mathbb{R} & \text { Regions } \\ \text { ar } \in \mathbb{A} \mathbb{R} & \text { Areas in regions }\end{array}$

Juan Gea-Bermúdez is with the Department of Management, Technical University of Denmark (DTU), Produktionstorvet, Bygning 426, 2800 Kongens Lyngby, Denmark (e-mail: jgeab@dtu.dk).

Kaushik Das and Matti Koivisto are with DTU Wind Energy, Technical University of Denmark, Ris $\varnothing$, Frederiksborgvej 399, 4000 Roskilde, Denmark.

Hardi Koduvere is with the Department of Electrical Power Engineering and Mechatronics, Tallinn University of Technology (TalTech).

\section{Subsets}

$\mathbb{S T O} \subset \mathbb{G} \mathbb{G} \quad$ Pure storage units

$\mathbb{G D} \subset \mathbb{G}$

$\mathbb{P T O H} \subset \mathbb{H O}$

$\mathbb{E L} \subset \mathbb{G} \mathbb{G} \mathbb{G}$ Dispatchable generation units

Electricity to heat generation units

Technologies delivering electricity to consumers

$\mathbb{H} \mathbb{E} \mathbb{T} \subset \mathbb{G} \mathbb{G} \mathbb{G}$ Technologies delivering heat to consumers

\section{Parameters \\ $F C_{g, a, y} \quad$ Installed input fuel consumption capacity [MW] \\ xloss Transmission loss [-] \\ $U S_{g, a, y, s, t}^{g e n} \quad$ Unit size of input fuel capacity of a generation unit [MW/unit] \\ $C^{\text {fom }} \quad$ Fixed annual cost [€] \\ $S L_{s} \quad$ Season length [days] \\ $M M T_{g} \quad$ Minimum maintenance time [days] \\ $D_{y, r, s, t}^{e l} \quad$ Exogenous gross electricity consumption rate [MW] \\ $D_{a, y, s, t}^{h} \quad$ Exogenous gross heat consumption rate [MW]}

\section{Positive decision variables}

$\begin{array}{ll}p_{g, a, y, s, t}^{e l} & \text { Net delivered electricity [MW] } \\ p_{g, a, y, s, t}^{h} & \text { Net delivered heat [MW] } \\ d_{g, a, y, s, t}^{f} & \text { Fuel consumption rate [MW] } \\ s t o l_{g, a, y, s, t} & \text { Storage loading rate [MW] } \\ x_{r, r^{\prime}, y, s, t} & \text { Transmission flow [MW] } \\ n_{g, a, y, s, t}^{a v, a, s, t} & \text { Units available for generation on [-] } \\ n_{g, a, y, s, t}^{n a v, p} & \text { Units not available for generation on mainte- } \\ \text { nance [-] } \\ n_{g, a, y, s, t}^{n a v} \text { Units not available for generation starting } \\ \text { maintenance [-] } \\ n_{g, a, y, s, t}^{n a v, s d} & \text { Units not available for generation stopping }\end{array}$

$\begin{array}{lc} & \text { maintenance [-] } \\ c_{g, a, y}^{v o m} & \text { Variable operational annual cost [€] } \\ c_{g, a, y}^{\mathrm{CO} O_{2}} & \text { Carbon dioxide tax annual cost }[€]\end{array}$

\section{INTRODUCTION}

Due to climate change and environmental concerns, energy systems including all energy vectors such as heating, transportation and agriculture, are converting to electricity-based energy usage. The European Commission has the vision of decarbonising the whole energy system by 2050 [1]. Denmark in this direction has the ambition to completely phase-out coal by 2030 [2]. Renewable based electricity generators such as hydro, wind, solar, or biomass are replacing the carbonbased generators. Many of these renewable energy sources 
are inherently variable in nature such as wind, solar, or microhydro. Consequently, increasing the share of such Variable Renewable Energy (VRE) sources in electricity systems increases the variability and uncertainty in the full energy system. Maintaining a stable and secure operation in the electricity system with large share of VRE can be very challenging for the power system operators. Major challenges involved in operational planning are estimation of operational reserves [3], or determining the ramp requirements and flexibility for the generators. This information is then used to mitigate the impact of variability in the electric power systems. Operational planning should include co-optimisation of all the sectors to avoid infeasibilities and sub-optimal solutions. For example, estimation of reserves from combined heat and power (CHP) units while only performing optimization of electrical power systems can create infeasibilities for the units in real-time due to heating constraints, and thereby, challenging the security of the system. Operational planning needs to be performed for long terms (at least one year) in order to determine adequacy of generation, quantification of reserves, planning of scheduled maintenance, hydrothermal scheduling, etc [4]. However, operational planning for large energy systems can have very high computational burden and large complexities. Performing these operational planning studies for short term can lead to infeasible solutions and is otherwise sub-optimal for reasons such as unable to consider long-term constraints, for example yearly schedule of hydro reservoirs.

In order to perform operational planning, unit commitment (UC) [5] or economic dispatch needs to be performed while optimizing all the energy sectors for long term. Many studies have analysed investment planning of energy systems [6], [7]. Schwele et. al. [6] demonstrated the value of UC for generation expansion planning as opposed to only using economic dispatch. Poncelet et. al. [7] developed generation expansion planning models considering flexibility and operating reserves. However, investment planning studies cannot capture all the details because of the computational limitations. All these studies only consider the stochasticity of generations and loads for few representative days or weeks. Therefore, these models are unable to capture all the possible scenarios and therefore, are likely not detailed enough for operational planning studies. Different UC modelling approaches have been researched for many years [5], [8], [9]. Mixed integer programming (MIP) is a widely used methodology for unit commitment. However, MIP based methods are computationally expensive. Lagrangian relaxation offers saving in computational time without compromising accuracy [10]. However, the application of such methods has been limited either to small test systems (for example, a 6 bus system in [11]) or to large systems but for small time intervals (24 hours in [12]) and limited amount of technologies involved (such as only for electrical systems). Recently, UC based studies have also been applied for multi-carrier energy systems but again the application has been limited to small test systems [13]. The integration of VRE has also been limited in the sense of modelling the details of weather dependencies. To the authors' knowledge, there is no research done on operational planning of large multicarrier energy systems for long-term studies (at least one year).
Therefore, there is a clear unmet need for research in this direction, where this paper contributes to.

This paper develops and evaluates a mathematical model to estimate and analyse different operational planning parameters (such as curtailment of VRE, energy prices, electricity imbalances for reserve estimation, revenue generated by different technologies and regions, inter-area tie line flows, congestion rent, etc.) for a very large energy system over a long period of time (one year) including detailed representation of renewable variations. The energy system comprising of all the North Sea countries for future scenarios (2020, 2030 and 2050) is developed and studied. The computational and practical challenges in modelling and implementation for such a large system are discussed. The computational cost and accuracy of results for different UC modelling approaches are compared.

The paper is structured as follows. Section II explains the mathematical model-based methodology applied to model the Day-Ahead (DA) market operation. Since the scale of the problem is large in terms of technologies, geography and time period, special considerations need to be taken to reduce computational complexity as described in Section III. Section IV presents the case study. Section V shows the results and discusses the limitations of the study while section VI summarizes the conclusions.

\section{Mathematical MODELling}

The methodology used in this paper to simulate the DA market can be split in four stages: DA optimisation (section II-A), VRE simulations (section II-B), storage and planned maintenance optimisation (section II-C), and stochastic outage simulations (section II-D). The stages are linked as shown in the flow chart of Figure 1. The sensitivity cases studied in this paper, which focus on the UC modelling approach, are presented in section II-E.

The optimisations and simulations, except for the VRE simulations, are performed with the energy system model Balmorel [14], an energy system tool, deterministic, open source [15], with a bottom-up approach. It has been traditionally used to model the electricity and district heating sectors, although it is currently being developed to increase its capabilities and include more sectors.

The temporal representation used is composed of years $\mathrm{y} \in$ $\mathbb{Y}$, which are disaggregated into seasons $s \in \mathbb{S}$ (in this paper days), which are composed of time steps $t \in \mathbb{T}$ (in this paper hours).

The geographical representation used is composed of countries, which are composed of regions $r \in \mathbb{R}$, which are disaggregated into areas $a \in \mathbb{A}$. Regions are built to represent copperplate zones for electricity transmission, whereas areas represent copperplate zones for heat transmission.

\section{A. Day-Ahead optimisation}

The DA optimisation of the energy markets is performed in a daily basis to replicate the behaviour of the spot market of electricity. This means that consecutive dispatch optimisations of 24 hours are performed. The results are linked from day to 


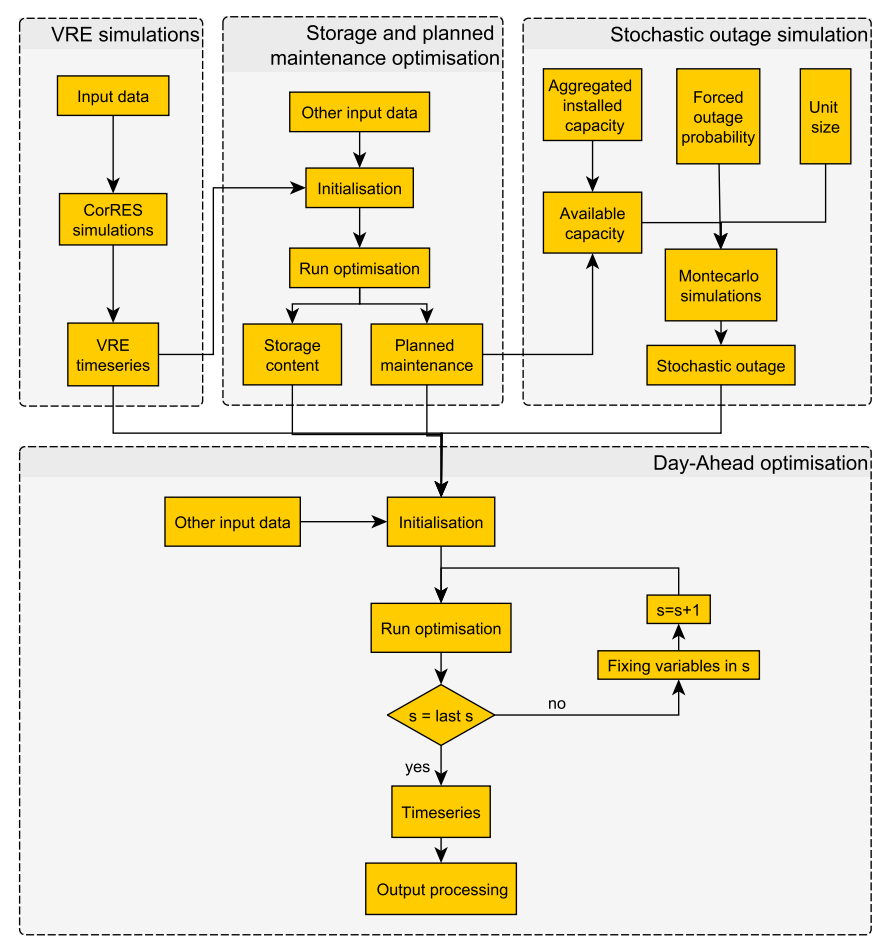

Fig. 1: Flow chart of the methodology.

day, which means that operational decisions made in previous days can have a limiting effect on the operation on the following days, depending on the flexibility of the units.

The storage content at the beginning of each day for the DA optimisation, as well as the planned maintenance, is fixed from the storage and planned maintenance optimisation. Additionally, the availability of the units within the day is also affected by stochastic outage, which together with the planned maintenance determine the final availability of the units to participate in the markets. Detailed mathematical formulation of the problem can be found in appendix A of [16]. Due to space limitation, only a few equations are shown here.

1) Objective function: The objective function constitutes of all operational costs of the studied system in the studied time steps and during the solving phase the value of the objective function is minimized (Equation 1). The time steps considered in this optimisation correspond to one day. In this paper, the costs have been aggregated into to variable operational and maintenance costs (vom), and emission tax costs (emi). Fixed operational and maintenance costs (fom) which depend on the installed capacity are also included in the objective function as a parameter. The disaggregation of these costs can be found in equations A2-A6 of [16].

$$
\min \sum_{y \in \mathbb{Y}} c_{y}^{v o m}+c_{y}^{e m i}+C_{y}^{f o m}
$$

2) System constraints: There are two main system constraints that need to be fulfilled in every time step. One of them is the electricity balance, in which the use of the electricity storage is optimized, and where most of the electricity demand is considered inelastic, except for the use of electricity to heat in district heating networks:

$$
\begin{array}{r}
\sum_{a \in \mathbb{A} R}\left(\sum_{g \in \mathbb{E L}} p_{g, a, y, s, t}^{e l}-\sum_{g \in \mathbb{S T O}, g \in \mathbb{E} \mathbb{L}} \text { stol }_{g, a, y, s, t}\right. \\
\left.-\sum_{g \in \mathbb{P} \mathbb{T} \mathbb{H}} d_{g, a, y, s, t}^{f}\right)=D_{r, y, s, t}^{e l} \\
+\sum_{r^{\prime} \in \mathbb{R}}\left(x_{r, r^{\prime}, y, s, t}-x_{r^{\prime}, r, y, s, t} \cdot(1-x \text { loss })\right) \\
\forall r \in \mathbb{R}, y \in \mathbb{Y}, s \in \mathbb{S}, t \in \mathbb{T}
\end{array}
$$

The other system constraint is the heat balance, where the use of heat storage is optimized and the heat demand considered inelastic:

$$
\begin{aligned}
& \sum_{g \in \mathbb{H} \mathbb{E} \mathbb{A}} p_{g, a, y, s, t}^{h}-\sum_{g \in \mathbb{S T} \mathbb{O}, g \in \mathbb{H} \mathbb{E} \mathbb{A} \mathbb{T}} \text { stol }_{g, a, y, s, t}=D_{a, y, s, t}^{h} \\
& \forall a \in \mathbb{A}, y \in \mathbb{Y}, s \in \mathbb{S}, t \in \mathbb{T}
\end{aligned}
$$

Ancillary services in the electricity sector were not included to simplify the problem.

3) Technological constraints: Generation technologies in the model are split in three categories: dispatchable generation units, pure storage units, and VRE units. Dispatchable technologies include electricity-only, Combined Heat and Power (CHP), boilers, heat pumps, geothermal, and hydroelectric power with reservoirs. These units are subject to several technical constraints. The operation of these technologies depends on the available units in each time step and specific technical characteristics of each type. The equations relevant for each of these technologies are described from A-29 to A-48 in the report [16]. Those storage units that do not receive inflow except from the active loading are defined as pure storage units. Examples of these technologies are hydro pumping, electric batteries, hot water tanks, or pit heat storage. The relevant equations for these units are equations A-49 to A71 in [16]. Non-dispatchable technologies included are solar $\mathrm{PV}$, solar heating, wind onshore and offshore, and hydro-runof-river. The equations limiting their production are A-72 and A-73 in [16]. Furthermore, electricity trade is allowed between regions, which are assumed to be copper plates, and is limited to the available transmission capacity between the regions in each time step. The relevant equation limiting their operation is A-74 in [16]. Heat trade between areas is not allowed.

\section{B. Modelling of renewable generation including fluctuations}

The CorRES model [17] is used for simulating the VRE generation time series used as inputs for Balmorel. CorRES is based on data from the Weather Research and Forecasting (WRF) model [18]. In addition to modelling the spatiotemporal dependencies in wind and solar PV generation, CorRES allows modelling of VRE technology development impacts on the VRE time series. For the presented case study, the assumed VRE technology developments towards 2050 are described in [19]. The resolution of the simulated VRE generation time series is hourly; data are aggregated to the regions used in Balmorel. 


\section{Storage and planned maintenance optimisation}

When simulating the DA market, it is important to capture that some of the decisions that market participants take are based on future expectations of market prices, rather than just planning for the next 24 hours. Planned maintenance and use of storage are part of these long-term decisions. In this paper, these two decisions are obtained by performing a full year dispatch optimisation. Planned maintenance and storage content at the beginning of each season are saved and forced in the DA optimisation. Planned maintenance is also used in the stochastic outage simulation to calculate the available capacity for production that can suffer an unexpected outage.

The formulation is similar to the one in section II-A, with a few exceptions. The time steps considered in this optimisation correspond to one year. All the equations can be found in [16]. In this paper only some equations are shown.

1) Available units: The availability of the units due to planned maintenance is endogenised in this optimisation. Planned maintenance decisions influence the availability factor of the units when simulating the DA market.

$$
\begin{array}{r}
\frac{F C_{g, a, y}}{U S_{g}^{g e n}}-n_{g, a, y, s}^{n a v, p m} \geq n_{g, a, y, s, t}^{a v, o n} \\
\forall g \in \mathbb{G D}, a \in \mathbb{A}, y \in \mathbb{Y}, s \in \mathbb{S}, t \in \mathbb{T}
\end{array}
$$

Additionally, the maximum number of units on maintenance is limited by the total number of units:

$$
\begin{array}{r}
\frac{F C_{g, a, y}}{U S_{g}^{g e n}} \geq n_{g, a, y, s}^{n a v, p m} \\
\forall g \in \mathbb{G D}, a \in \mathbb{A}, y \in \mathbb{Y}, s \in \mathbb{S}, t \in \mathbb{T}
\end{array}
$$

2) Yearly maintenance requirement: The following equation make sure the minimum maintenance time (MMT) per technology is respected in each year:

$$
\begin{aligned}
\sum_{s \in \mathbb{S}} n_{g, a, y, s}^{n a v, p m, s u} \cdot S L_{s} & =M M T_{g} \cdot \frac{F C_{g, a, y}}{U S_{g}^{g e n}} \\
\forall g & \in \mathbb{G D}, a \in \mathbb{A}, y \in \mathbb{Y}
\end{aligned}
$$

3) Uninterrupted maintenance: Maintenance is assumed to take place uninterrupted, i.e. in consecutive seasons:

$$
\begin{array}{r}
\sum_{s^{\prime}=1}^{M M T_{g}} n_{g, a, y, s-s^{\prime}}^{n a v, p m, s u} \leq n_{g, a, y, s}^{n a v, p m} \\
\forall g \in \mathbb{G} \mathbb{G} \mathbb{G}, a \in \mathbb{A}, y \in \mathbb{Y}, s \in \mathbb{S}
\end{array}
$$

4) Logical conditions: The number of units on maintenance depends on the units starting or stopping maintenance:

$$
\begin{array}{r}
n_{g, a, y, s}^{n a v, p m}-n_{g, a, y, s-1}^{a v, p m}=n_{g, a, y, s}^{n a v, s u}-n_{g, a, y, s}^{n a v, s d} \\
\forall g \in \mathbb{G} \mathbb{G} \mathbb{G}, a \in \mathbb{A}, y \in \mathbb{Y}, s \in \mathbb{S}
\end{array}
$$

In order to reflect the discrete nature of the generation units that are part of the energy system, the following variables are restricted to be integer variables. As mentioned in section III-B, this constraint is relaxed however due to computational complexity in the optimisation.

$$
\begin{array}{r}
n_{g, a, y, s, t}^{a v, o n}, n_{g, a, y, s}^{n a v, p m}, n_{g, a, y, s}^{n a v, p m, s u}, n_{g, a, y, s}^{n a v, p m, s d} \in \mathbb{Z}^{+} \\
\forall g \in \mathbb{G} \mathbb{G} \mathbb{G}, a \in \mathbb{A}, y \in \mathbb{Y}, s \in \mathbb{S}, t \in \mathbb{T}
\end{array}
$$

\section{Stochastic outage simulations}

Unexpected operational problems can lead to making units unavailable until the problem is fixed, which can influence market prices. Hence, it is relevant to capture these occurrences. Using as input parameters the total capacity of a unit type in each area, the size of a single unit, the planned maintenance (if previously calculated), and the probability of suffering an outage, Monte carlo simulations are performed for each time step and unit in the system to simulate these outages. The outcome from these simulations is then fed to the DA optimisation, and the relevant variables fixed. This approach is applied to all units excepts to VRE ones, since their availability is part of the time series used. The formulation can be found in equations A-85 and A-86 of [16].

\section{E. Sensitivity cases: unit commitment modelling approaches}

To analyse the importance of the UC modelling approach when modelling the DA market, three different sensitivity cases of UC modelling approaches are studied in the DA optimisations: 1) adding UC constraints with integer commitment variables (UC-MIP), 2) adding UC constraints with relaxed commitment variables (UC-RMIP), and 3) not adding constraints nor corresponding commitment variables (NO$\mathrm{UC})$.

\section{SPECIAL CONSIDERATIONS FOR LONG-TERM OpERATIONAL PlanNing OF LARGE-SCALE ENERGY SYSTEM}

\section{A. Unit commitment assumptions}

Introducing UC in the optimisation allows for an improved representation of conventional generation, at the cost of increasing considerably computational complexity due to the use integer variables. Solving a large-scale MIP problem can be intractable. To deal with this problem, one can either relax the integer variables, or limit the technologies modelled with integer variables. In this paper, the second approach is considered so the impact of the different optimisation approaches can be evaluated. The technologies modelled with UC integer variables are almost all type of fuel-based thermal plants, i.e. gas turbines, steam turbines, combined cycle turbines and boilers. Engines were not included since they are very fast and their size is generally much smaller than other generators, making their impact negligible. The rest of the technologies, i.e. hydro reservoirs, other storage, $\mathrm{P} 2 \mathrm{H}$, and VRE, were not modelled with UC variables to reduce the complexity of the problem.

\section{B. Simplifications in storage and planned maintenance opti- misation}

Optimal planning of maintenance is solved as a Relaxed Mixed Integer Problem (RMIP), including all days of the years but with 1 every 3 hours to reduce complexity. Planned maintenance is only computed for the units modelled with UC costs and variables (see section III-A). 


\section{Case Study: The North Sea offshore Grid}

The study case used in this paper corresponds to the offshore grid scenario presented in [19], developed as part of the North Sea Offshore Network - Denmark project. The scenario focuses on the following countries: Norway, Great Britain, Netherlands, Belgium, and Germany. The sectors included are the electric and district heating sectors. The study case shows towards 2050 a high share of VRE, transmission interconnection, and low sector coupling in the countries in focus. The capacity development was highly influenced by the assumptions on increasing $\mathrm{CO}_{2}$ EU ETS price: 5.93, 75.16, and $127.772015 € /$ ton in 2020, 2030, and 2050 respectively. More details about the scenario can be read in [19], [20], and [21].

\section{RESUlTS AND DISCUSSION}

First, section V-A focuses on key results obtained from the storage and planned maintenance optimisations. Section $\mathrm{V}$-B presents the results from the Day-Ahead optimisations, focusing on the influence of VRE penetration and the UC modelling approach. The limitations of the study are discussed in section V-C. Costs and prices are in $€ 2012$.

\section{A. Storage and planned maintenance optimisation}

1) Planned maintenance: The installed capacity under planned maintenance of district heating boilers burning waste in the countries in focus is shown in Figure 2. Most of the planned maintenance takes place during summer, which is when the district heat demand is lowest. By 2050 there is more maintenance in May and September, which could be linked to stronger sector coupling between the electricity and heat sector.

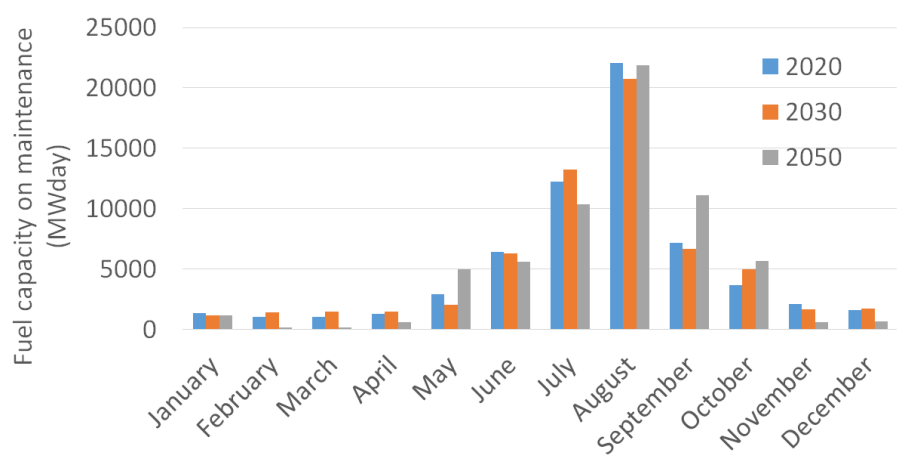

Fig. 2: District heating boilers burning waste on planned maintenance per month in the countries in focus (MWday).

2) Planned storage use: The aggregated planned energy content along the year of hydro reservoirs in Norway is shown in Figure 3. The minimum value of each profile has been subtracted. The results show that reservoirs are mainly filled during the summer and discharged during winter. The maximum energy content is higher in 2020 than in 2030 and 2050. This is a result of storing less energy in the reservoir during the year, which is linked to using hydro energy for balancing VRE. These results strengthen the importance of performing full year optimisations.

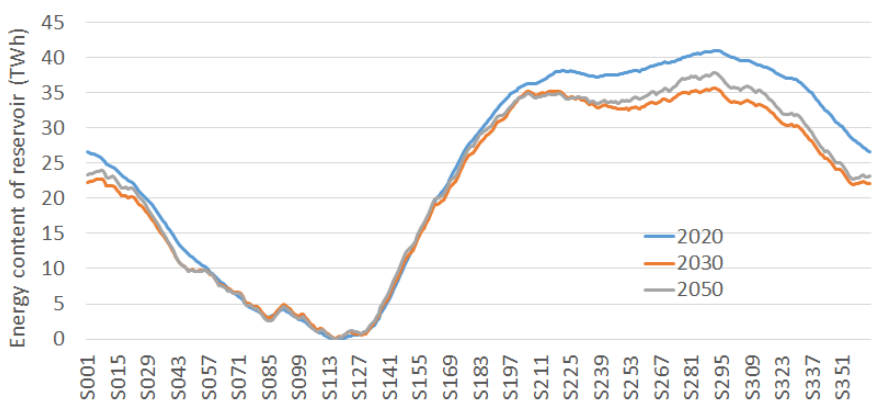

Fig. 3: Planned energy content along the year of hydro reservoirs in Norway (TWh). The minimum value of each profile has been subtracted.

\section{B. Day-Ahead optimisation}

1) Annual production: The aggregated generation of electricity and heat per year, scenario, and technology in the countries in focus for different UC approaches is depicted in Figure 4. The penetration of VRE in the electric sector towards 2050 is remarkable, at the expense of decreasing the use of thermal technologies. The results show that the share of $\mathrm{CO}_{2}$ free generation increases from $64 \%$ in 2020 to $91 \%$. On the heating side, partial electrification is observed towards 2050 , although the majority of district heat is still delivered with CHP units. Introducing UC constraints increases slightly the aggregated production of thermal power units and reduces VRE generation.

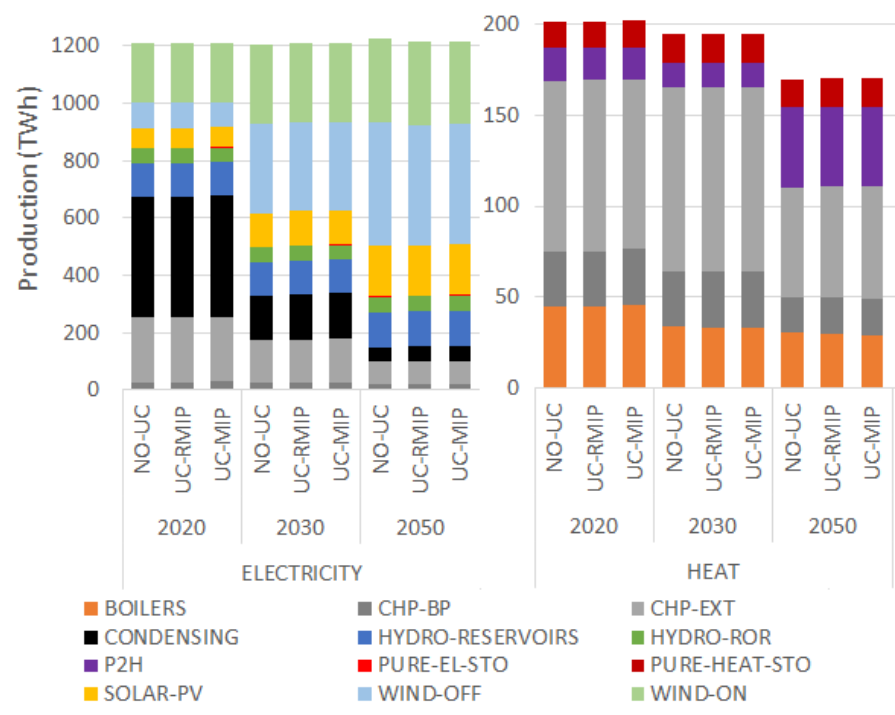

Fig. 4: Production development per commodity, year, technology type, and UC approach (TWh).

The average capacity factor of thermal power units for electricity with/without heat generation is shown in Figure 5 for the UC-MIP approach. The difference with NO-UC and UC-RMIP is negligible. The electricity capacity factor is calculated with rated electricity generation capacity, whereas the heat one uses rated heat generation capacity. The results show that towards 2050, the average electricity capacity factor decreases for CHP back pressure units (CHP-BP), 
TABLE I: Disaggregated operational costs (billion $€$ ).

\begin{tabular}{|c|c|c|c|c|c|}
\hline \multirow{2}{*}{ Year } & $\begin{array}{c}\text { Modelling } \\
\text { approach }\end{array}$ & $\begin{array}{c}\text { Fixed } \\
\text { costs }\end{array}$ & $\begin{array}{c}\text { Variable } \\
\text { costs }\end{array}$ & $\begin{array}{c}\text { Emission } \\
\text { costs }\end{array}$ & Total \\
\hline \multirow{2}{*}{$\mathbf{2 0 2 0}$} & NO-UC & 8.2 & 12.3 & 1.8 & 22.4 \\
\cline { 2 - 6 } & UC-RMIP & 8.2 & 13.6 & 1.9 & 23.7 \\
\cline { 2 - 6 } & UC-MIP & 8.2 & 14.1 & 1.9 & 24.2 \\
\hline \multirow{2}{*}{2030} & NO-UC & 9.6 & 13.5 & 6.3 & 29.4 \\
\cline { 2 - 6 } & UC-RMIP & 9.6 & 14.4 & 6.5 & 30.5 \\
\cline { 2 - 6 } & UC-MIP & 9.6 & 14.7 & 6.6 & 30.9 \\
\hline \multirow{2}{*}{$\mathbf{2 0 5 0}$} & NO-UC & 10.6 & 8.5 & 6.1 & 25.2 \\
\cline { 2 - 6 } & UC-RMIP & 10.6 & 9.0 & 6.2 & 25.8 \\
\cline { 2 - 6 } & UC-MIP & 10.6 & 9.1 & 6.3 & 26.0 \\
\hline
\end{tabular}

CHP extraction units (CHP-EXT) and electricity-only units (CONDENSING), especially for the last two types. The heat capacity factor also tends to decrease. These results suggest that massive penetration of VRE might challenge the profitability of thermal units towards 2050 .

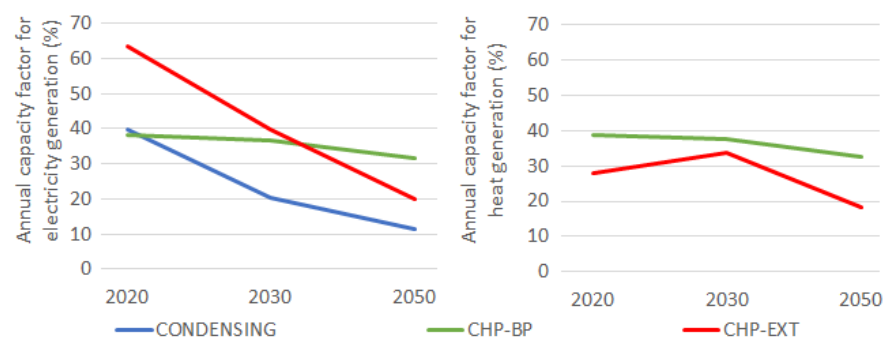

Fig. 5: Average capacity factor development of thermal power units for electricity (left) and heat (right) generation using UCMIP. The difference with NO-UC and UC-RMIP is negligible.

2) System costs: The disaggregated operational costs development in the countries in focus per UC approach is shown in Table I. The total operational costs increase in 2030 with respect to 2020, and decrease in 2050 compared to 2030. This development is linked to VRE penetration and $\mathrm{CO}_{2}$ tax assumptions. By 2030, there is still considerable fossil generation, leading to high $\mathrm{CO}_{2}$ tax costs. By 2050, even though the $\mathrm{CO}_{2}$ tax is higher than in 2030, there is much less fossil generation, which together with further VRE penetration leads to considerably less variable costs. Using UC-RMIP leads to much closer results to UC-MIP than using NO-UC, although the difference decreases towards 2050, again due to less fossil generation. Not including UC constraints, i.e. using NO-UC, underestimates both variable and $\mathrm{CO}_{2}$ costs since it overestimates the flexibility of the units when ignoring relevant costs such as start-up.

3) Electricity prices: The influence of the UC approach in the cumulative probability curves for the electricity prices in each scenario and year, for the region DK1 is shown in Figure 6. The influence of the optimisation approach decreases towards 2050, which is explained with the decrease of thermal power capacity and use towards 2050 , since they are the ones affected by the different UC approaches. UC-MIP leads to overall higher prices in all price-range due to forcing discrete
TABLE II: Influence of UC approach in aggregated yearly curtailment per technology type in the countries in focus. Difference with respect to UC-MIP approach (TWh).

\begin{tabular}{|c|c|c|c|c|c|c|}
\hline \multirow{2}{*}{ Technology type } & \multicolumn{3}{|c|}{ NO-UC } & \multicolumn{3}{c|}{ UC-RMIP } \\
\cline { 2 - 7 } & $\mathbf{2 0 2 0}$ & $\mathbf{2 0 3 0}$ & $\mathbf{2 0 5 0}$ & $\mathbf{2 0 2 0}$ & $\mathbf{2 0 3 0}$ & $\mathbf{2 0 5 0}$ \\
\hline Wind offshore & -0.9 & -4.3 & -13.9 & 0 & 0 & 0 \\
\hline Wind onshore & -0.3 & 0 & -0.1 & 0 & 0 & 0 \\
\hline Total & -1.2 & -4.3 & -14 & 0 & 0 & 0 \\
\hline
\end{tabular}

block sizes to be on/off for thermal units. The number of hours with very low prices (where VRE curtailment sets the price), are higher with UC-RMIP, and especially with UC-MIP. These low prices generally correspond to those hours where it is cheaper (or the only feasible way) to increase the use of more expensive generation units, rather than starting/shutting them. In the mid-range prices, the prices for UC-RMIP tend to be the lowest, which can be explained in a similar way to VRE curtailment, but instead, what it is being "curtailed" is the next available cheaper generator. On the high-range prices, NOUC underestimates high prices, which can be explained with the non-consideration of restrictive constraints like minimumon/off time, minimum production, and ramping. The order of magnitude of the prices is highly influenced by the $\mathrm{CO}_{2}$ tax assumption development (section IV).

4) Curtailment: The influence of the UC approach on curtailment for different technology types in the countries in focus per year are shown in Table II. Not considering UC costs leads to less curtailment. On the other hand, wind offshore is curtailed more often than onshore due to operation costs assumptions, i.e. variable costs for offshore are more expensive than for onshore. The impact of not considering UC costs on curtailment increases towards 2050, when there is higher VRE penetration.

5) Average revenue of wind and solar PV units: The influence of the UC approach on the variability of the average revenue per energy unit sold in each region and year from the operation in the DA market for wind and solar PV units is shown in Figure 7. The results show a decrease in the variability of the average revenue across countries towards 2050, mainly due to grid expansion, which remarks the importance of large-scale energy system analysis. Solar PV's average revenue is higher in 2020 and 2030 than wind unit's, but slightly smaller in 2050. With a UC-MIP approach, average revenues are slightly higher for both technologies. The yearly levels of average revenue are directly linked to the DA price development.

6) Electricity-only thermal plant operation: The influence of the UC approach in the utilization factor of electricityonly thermal generation units using natural gas or nuclear fuels is shown in Table III. The results show a decrease in utilization for all the considered units towards 2050. Units burning natural gas half their capacity factor with respect to 2020 in 2030 and stay almost constant by 2050. Nuclear power capacity factor also decreases considerably towards 2050 . The 


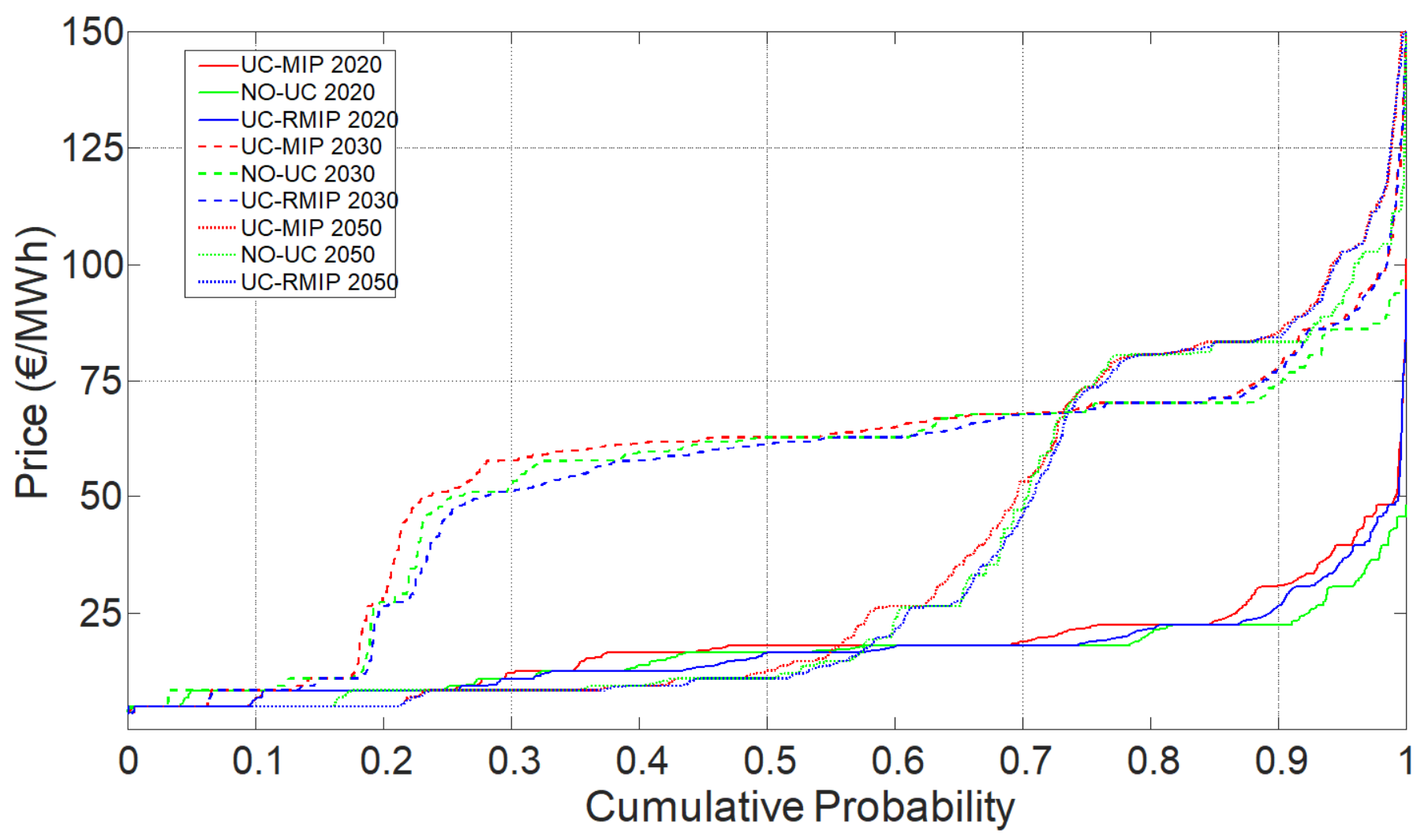

Fig. 6: Probability distribution function of the hourly electricity price in DK1 for each year and UC modelling approach.

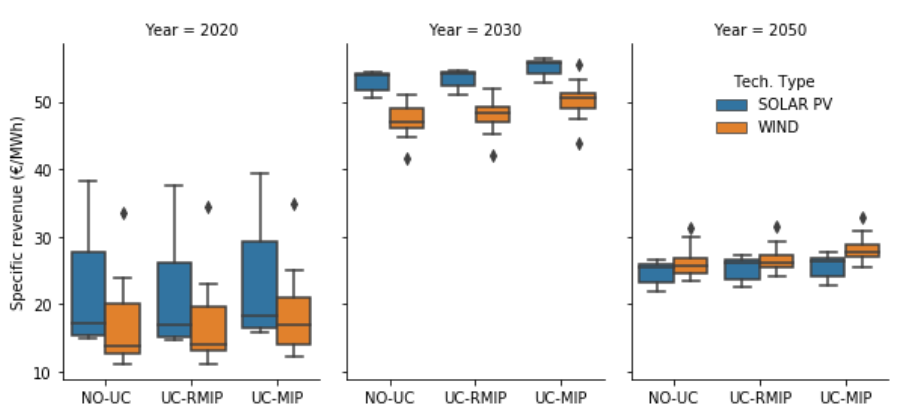

Fig. 7: Regional variability of the average revenue per energy unit sold of wind and solar PV units in DA market towards 2050. Influence of UC modelling approach.

TABLE III: Influence of UC modelling approach and year in annual capacity factor of electricity-only thermal generation units for different fuels.

\begin{tabular}{|c|c|c|c|c|c|c|}
\cline { 2 - 7 } \multicolumn{1}{c|}{} & \multicolumn{3}{c|}{ NATURAL GAS } & \multicolumn{3}{c|}{ NUCLEAR } \\
\hline Modelling approach & $\mathbf{2 0 2 0}$ & $\mathbf{2 0 3 0}$ & $\mathbf{2 0 5 0}$ & $\mathbf{2 0 2 0}$ & $\mathbf{2 0 3 0}$ & $\mathbf{2 0 5 0}$ \\
\hline NO-UC & 17.1 & 7.6 & 7.6 & 95.2 & 85.5 & 55.1 \\
\hline UC-RMIP & 18.0 & 8.6 & 7.7 & 93.5 & 87.0 & 61.5 \\
\hline UC-MIP & 19.7 & 9.2 & 8.0 & 94.8 & 87.4 & 62.6 \\
\hline
\end{tabular}

UC approach impacts the utilization of nuclear power by 2050 , overestimating its flexibility when using NO-UC. These results are influenced by the decrease of nuclear power in the system towards 2050, and the increasing penetration of VRE.

The impact of the UC modelling approach on hourly nuclear operation, highly affected by UC constraints, is shown in
Figure 8, which shows the hourly operation of nuclear power plants for four consecutive days in Great Britain. The figure depicts that towards 2050, with higher VRE penetration, unless UC costs and constraints are considered, nuclear units start up and shut down with high frequency, which might be extremely challenging, and maybe unrealistic. These results strengthen the importance of multiple scenario year analysis as well as the UC modelling approach used.

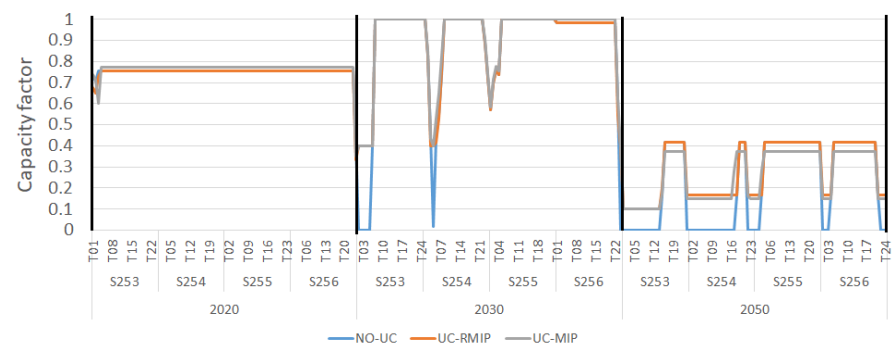

Fig. 8: Hourly capacity factor of nuclear power in four consecutive days in Great Britain in different scenario years. Impact of UC modelling approach.

7) Computational time: The influence of the UC modelling approach on the average computational time of the optimisation for a simulation of a day is shown in Table IV. The computational time required to solve the UC-MIP case is much higher than the others, which decreases significantly towards 2050 since the number of thermal power plants is considerably reduced in this scenario. Using UC-RMIP leads 
TABLE IV: Influence of year and UC modelling approach on average computational time for a simulation of a day (seconds) ${ }^{1}$.

\begin{tabular}{|c|c|c|c|c|}
\hline Modelling approach & $\mathbf{2 0 2 0}$ & $\mathbf{2 0 3 0}$ & $\mathbf{2 0 5 0}$ & Average \\
\hline NO-UC & 3.47 & 4.55 & 3.21 & 3.74 \\
\hline UC-RMIP & 30.29 & 51.04 & 44.56 & 41.96 \\
\hline UC-MIP & 293.89 & 264.93 & 152.51 & 237.11 \\
\hline
\end{tabular}

to closer computational times to NO-UC than to UC-MIP.

\section{Limitations of the study}

The simplifications undertaken to reduce the complexity of the problem limit the findings of this study. The flexibility of technologies for which UC constraints were not applied could have been overestimated, especially for hydro power units. Furthermore, performing the analysis with several weather years and/or performing stochastic optimisation would help understand the role of uncertainty in the results. In this paper, a unique weather year was used for simplification (2012). Adding ancillary services would have also impacted the results, especially prices, affected by must-run operation.

\section{Vi. Conclusions}

This paper proposes a method to perform optimal operational planning of large-scale energy systems with high penetration of renewable energy. Furthermore, it analyses the influence of UC modelling on the results. The results highlight the importance of analysing multiple scenario years with long time series, including several sectors, as well as the value of not restricting the analysis to small scale. The results for the studied case show how the penetration of VRE towards 2050 challenges thermal units' traditional operation in the electricity and district heating sectors towards 2050. Including UC constraints with integer variables leads to more realistic behaviour of the units, at the cost of increasing considerably the computational time. Relaxing integer variables reduces significantly the computational time, but medium-level prices are underestimated. Not including UC constraints leads to underestimation of costs, VRE curtailment, VRE's average revenue per energy unit sold, as well as price volatility. It also overestimates the flexibility of the thermal units. Hence, depending on the purpose of the analysis, it is recommended to think carefully on which UC modelling approach to use and acknowledge the limitations. When the focus is on prices and revenues, using unit commitment constraints with integer variables is preferable, otherwise, relaxing the integer variables is encouraged.

\section{ACKNOWLEDGEMENT}

This paper is a part of NSON-DK and Flex4RES projects funded by the Danish Energy Agency, EUDP (grant 640180032; previously ForskEL) and Nordic Energy Research (grant

\footnotetext{
${ }^{1}$ In UC-MIP runs, a relative gap of 0.00001 , and time limit of 660 seconds was assumed. The solver used was CPLEX LP1. The optimisations are performed in High Performance Computing clusters (10 nodes were used). More information on https://www.hpc.dtu.dk/ page $_{i} d=2520$
}

76084), respectively. Matti Koivisto acknowledges support from the PSfuture project (La Cour Fellowship, DTU Wind Energy). The authors thank Hans Ravn for his contributions.

\section{REFERENCES}

[1] European Commission, "2050 long-term strategy." [Online]. Available: https://ec.europa.eu/clima/policies/strategies/2050_en\#tab-0-0

[2] Energi-,Forsynings- og Klimaministeriet, "Denmark's Draft Integrated National Energy and Climate Plan." [Online]. Available: https://ens.dk/sites/ens.dk/files/EnergiKlimapolitik/ denmarks_draft_integrated_energy_and_climate_plan.pdf

[3] K. Das, M. Litong-Palima, P. Maule, M. Altin, A. D. Hansen, P. E. Sørensen, and H. Abildgaard, "Adequacy of frequency reserves for high wind power generation," IET Renewable Power Generation, vol. 11, no. 8, pp. 1286-1294, 2016.

[4] "Current issues in operational planning (of power systems)," IEEE Transactions on Power Systems, vol. 7, no. 3, pp. 1197-1210, 1992.

[5] N. P. Padhy, "Unit commitment-a bibliographical survey," IEEE Transactions on power systems, vol. 19, no. 2, pp. 1196-1205, 2004.

[6] A. Schwele, J. Kazempour, and P. Pinson, "Do unit commitment constraints affect generation expansion planning? a scalable stochastic model," Energy Systems, pp. 1-36, 2019.

[7] K. Poncelet, E. Delarue, and W. D'haeseleer, "Unit commitment constraints in long-term planning models: Relevance, pitfalls and the role of assumptions on flexibility," Applied Energy, vol. 258, p. 113843, 2020.

[8] F. Zhuang and F. D. Galiana, "Towards a more rigorous and practical unit commitment by lagrangian relaxation," IEEE Transactions on Power Systems, vol. 3, no. 2, pp. 763-773, 1988.

[9] A. Frangioni, C. Gentile, and F. Lacalandra, "Tighter approximated milp formulations for unit commitment problems," IEEE Transactions on Power Systems, vol. 24, no. 1, pp. 105-113, 2008.

[10] T. Li and M. Shahidehpour, "Price-based unit commitment: A case of lagrangian relaxation versus mixed integer programming," IEEE transactions on power systems, vol. 20, no. 4, pp. 2015-2025, 2005.

[11] C. Zhao and R. Jiang, "Distributionally robust contingency-constrained unit commitment," IEEE Transactions on Power Systems, vol. 33, no. 1, pp. 94-102, 2017.

[12] J. Shi and S. S. Oren, "Stochastic unit commitment with topology control recourse for power systems with large-scale renewable integration," IEEE Transactions on Power Systems, vol. 33, no. 3, pp. 3315-3324, 2017.

[13] M. Yan, N. Zhang, X. Ai, M. Shahidehpour, C. Kang, and J. Wen, "Robust two-stage regional-district scheduling of multi-carrier energy systems with a large penetration of wind power," IEEE Transactions on Sustainable Energy, vol. 10, no. 3, pp. 1227-1239, 2018.

[14] F. Wiese et al., "Balmorel open source energy system model," Energy Strategy Reviews, vol. 20, pp. 26-34, 2018. [Online]. Available: https://doi.org/10.1016/j.esr.2018.01.003

[15] Github, "Balmorel model." [Online]. Available: https://github.com/ balmorelcommunity/Balmorel

[16] P. Kanellas, K. Das, J. Gea-Bermúdez, and P. Sørensen, "Balancing Tool Chain : Balancing and automatic control in North Sea Countries in 2020, 2030 and 2050," DTU Wind Energy, 2020. [Online]. Available: https://orbit.dtu.dk/en/publications/balancing-toolchain-balancing-and-automatic-control-in-north-sea

[17] M. Koivisto, K. Das, F. Guo, P. Sørensen, E. Nuño, N. Cutululis, and P. Maule, "Using time series simulation tool for assessing the effects of variable renewable energy generation on power and energy systems," WIREs Energy and Environment, vol. 8, 2019.

[18] W. Skamarock and J. Klemp and J. Dudhia and D. Gill and D. Barker and M. Duda and X. Huang and W. Wang and J. Powers, "Description of the advanced research wrf version 3," Boulder, Colorado, USA.

[19] M. Koivisto, J. Gea-Bermúdez, and P. Sørensen, "North sea offshore grid development: Combined optimization of grid and generation investments towards 2050," IET Renewable Power Generation, 2019. [Online]. Available: https://doi.org/10.1049/iet-rpg.2019.0693

[20] J. Gea-Bermúdez, K. Das, L. Pade, M. Koivisto, and P. Kanellas, "NSON-DK Day-Ahead market operation analysis in the North Sea region towards 2050," 2019. [Online]. Available: https://orbit.dtu.dk/en/publications/nson-dk-day-aheadmarket-operation-analysis-in-the-north-sea-regi

[21] J. Gea-Bermúdez, L. Pade, M. Koivisto, and H. Ravn, "Optimal generation and transmission development of the north sea region: Impact of grid architecture and planning horizon," Energy, vol. 191, 2020. 